\title{
Analysis of PD-L1, T-cell infiltrate and HLA expression in chondrosarcoma indicates potential for response to immunotherapy specifically in the dedifferentiated subtype
}

\author{
Marie Kostine ${ }^{1,2}$, Arjen HG Cleven ${ }^{1}$, Noel FCC de Miranda ${ }^{1}$, Antoine Italiano ${ }^{3}$, \\ Anne-Marie Cleton-Jansen ${ }^{1}$ and Judith VMG Bovée ${ }^{1}$ \\ ${ }^{1}$ Department of Pathology, Leiden University Medical Center, Leiden, The Netherlands; ${ }^{2}$ Department of \\ Rheumatology, Hôpital Pellegrin, Centre Hospitalier Universitaire de Bordeaux, Bordeaux Cedex, France and \\ ${ }^{3}$ Department of Medical Oncology, Institut Bergonié, Bordeaux Cedex, France
}

\begin{abstract}
Therapies targeting the programmed cell death 1 (PD-1) or its ligand (PD-L1) promote antitumor T-cell activity, leading to unprecedented long-lasting tumor responses in some advanced cancers. Because of radiotherapy and chemotherapy resistance, no effective treatments have been defined for advanced chondrosarcomas. We here report an immunohistochemical analysis of PD-L1 expression in a large series of conventional, mesenchymal, clear cell and dedifferentiated chondrosarcomas using tissue microarrays. In the PD-L1-positive tumors, we analyzed the immune microenvironment (T-cell and macrophage infiltration as well as HLA class I expression) using whole sections. PD-L1 expression was absent in conventional $(n=119)$, mesenchymal $(n=19)$ and clear cell $(n=20)$ chondrosarcomas. Forty-one percent $(9$ of the 22) of dedifferentiated chondrosarcomas displayed PD-L1 positivity. These results were confirmed in an independent cohort using whole tissue sections of dedifferentiated chondrosarcomas in which PD-L1 expression was detected in 52\% (11 of the 21) of cases. PD-L1 expression was exclusively found in the dedifferentiated component and expression positively correlated with other immune parameters such as high number of tumor-infiltrating lymphocytes $(P=0.014)$ and positive HLA class I expression $(P=0.024)$ but not with patient overall survival $(P=0.22)$. The presence of PD-L1 expression in association with immune-infiltrating cells and HLA class I expression in nearly $50 \%$ of the dedifferentiated chondrosarcomas provides rationale for including these patients in clinical trials with PD-1/PD-L1-targeted therapies.
\end{abstract}

Modern Pathology (2016) 29, 1028-1037; doi:10.1038/modpathol.2016.108; published online 17 June 2016

Chondrosarcomas constitute a malignant group of cartilaginous matrix-producing neoplasms, with diverse morphological features and clinical behavior. ${ }^{1}$ Conventional chondrosarcoma is the second most common primary bone malignancy after osteosarcoma and can be categorized according to their location into central (85\%) and peripheral chondrosarcomas $(15 \%)$. Their histology is similar, with atypical chondrocytes and abundant extracellular matrix, but they differ at the genetic level. Notably, mutations in isocitrate dehydrogenase

Correspondence: Dr JVMG Bovée, MD, PhD, Department of Pathology, Leiden University Medical Center, Albinusdreef 2, Leiden 2333 ZA, The Netherlands.

E-mail: j.v.m.g.bovee@lumc.nl

Received 19 March 2016; revised 27 April 2016; accepted 1 May 2016; published online 17 June 2016
(IDH) 1 and 2 are found in approximately half of conventional central chondrosarcomas while mutations in genes encoding exostosin (EXT) 1 and 2 characterize peripheral chondrosarcomas. ${ }^{2,3}$ Histological grade is strongly correlated with clinical prognosis with a 10-year survival rate of $83 \%$ for atypical cartilaginous tumor/grade I but only $29 \%$ for grade III. In addition to conventional chondrosarcoma, several rare subtypes of chondrosarcoma are discerned (dedifferentiated, mesenchymal and clear cell), together constituting $10-15 \%$ of all chondrosarcomas. Clear cell chondrosarcoma is a low-grade malignant tumor with a 10-year survival rate reported around $90 \%$ while dedifferentiated and mesenchymal chondrosarcoma are both highly malignant, with frequent occurrence of distant metastases and $<30 \%$ survival at 5 and 10 years, respectively. ${ }^{4}$ IDH mutations are also present in around half of the 
dedifferentiated chondrosarcomas. ${ }^{5}$ Wide surgical excision remains the best available treatment for chondrosarcoma patients, and in case of nonresectable or metastatic disease, effective options are limited owing to the relative radiotherapy and chemotherapy resistance of these tumors.

The recent approval of ipilimumab, an anticytotoxic T-lymphocyte-associated antigen 4 (CTLA-4) antibody, followed by nivolumab and pembrolizumab, two anti-programmed death 1 (anti-PD-1) antibodies, represented a considerable progress in the treatment of metastatic melanoma and non-small cell lung cancer. ${ }^{6-9}$ By enhancing antitumor T-cell activity, some unprecedented long-lasting tumor responses were observed in patients with unresectable or metastatic disease. The clinical value of these immune checkpoint inhibitors is currently being investigated in various solid tumors and hematological malignancies. Their expression and role in sarcomas is not yet well characterized but is currently under investigation. ${ }^{10}$ However, to date there is scarce data regarding the immune microenvironment and the presence of the immune checkpoint PD-1 with its ligand PD-L1 in chondrosarcoma. Recently, a preclinical study revealed the absence of PD-L1 expression in six mesenchymal chondrosarcomas. ${ }^{11}$ This may suggest that this chondrosarcoma subtype is less suited for PD-1/ PD-L1 blockade but these observations cannot be generalized to all chondrosarcomas.

PD-L1 expression, either by the tumor cells themselves or by the tumor-associated immune cells, can be an effective mechanism for tumors to evade immune-mediated destruction. ${ }^{12}$ A high mutational load in tumors and the presence of a spontaneous antitumor immune reaction prior to treatment have been associated with predicting better response to both CTLA-4 and PD-1 blockade. ${ }^{13-15}$ Whole-exome sequencing of 49 chondrosarcomas identified a median of 22 non-synonymous mutations per tumor (range 1-93). ${ }^{16}$ Grade II, grade III and dedifferentiated chondrosarcomas were significantly associated with a higher mutation burden, which can indicate a potential sensitivity to immune checkpoint blockade. Another reported predictive factor for response to anti PD-1 agents is PD-L1 expression before treatment, but this remains a matter of debate owing to the dynamic process of PD-L1 expression, the multiplicity of detection methods and the use of different cutoff values. ${ }^{17}$ Moreover, the predictive value of PD-L1 expression also differs between tumor subtypes and the monoclonal antibody used. As an example, PD-L1 status alone does not seem to be useful in the selection of melanoma patients for nivolumab. On the other hand, when its expression was retrospectively determined in non-small cell lung cancers, it identified patients with an enhanced likelihood to benefit from pembrolizumab. ${ }^{18-20}$

Cytotoxic $\mathrm{T}$ cells are the main executioners of antitumor immunity induced by these immune checkpoint blockers but require antigenic presentation in the context of human leukocyte antigen (HLA) molecules. Loss or inability to upregulate HLA class I expression is a common mechanism of tumor immune escape to avoid the T-cell recognition. Therefore, assessing HLA class I expression on tumor cells might be an important prerequisite for the success of T-cell-mediated immunotherapies. ${ }^{21}$

In order to evaluate whether chondrosarcoma patients are eligible for PD-1- or PD-L1-targeted therapies, we determined the PD-L1 expression and its potential association with HLA class I expression and immune-infiltrating cells in a large series of chondrosarcomas by using immunohistochemistry.

\section{Materials and methods}

\section{Patient Material}

Two tissue microarrays were used for which clinicopathological data were reported in detail previously. ${ }^{22,23}$ Briefly, primary tumors of 137 patients with conventional chondrosarcomas (central chondrosarcoma, $n=92$; peripheral chondrosarcoma, $n=45$ ) and 20 benign cartilage tumors (osteochondroma, $n=9$; enchondroma, $n=11$ ) were included in a conventional tissue microarray while dedifferentiated chondrosarcomas $(n=25)$, mesenchymal chondrosarcomas $(n=21)$ and clear cell chondrosarcomas $(n=20)$ from five institutes (Leiden, Oxford, Bologna, Budapest and Graz) were collected in a rare subtype tissue microarray.

For the validation cohort, we retrieved 23 formalinfixed, paraffin-embedded tissue blocks from 22 patients with dedifferentiated chondrosarcoma (2009-2015) from the archives of the Pathology Department of the Leiden University Medical Center. We used whole sections to assess PD-L1, immune infiltrate and HLA class I expression in these tumors. Nineteen tissue samples were primary tumors, three were metastases and one was a local relapse. For one patient, material from both primary tumor and metastasis was available. Diagnoses were confirmed by an experienced bone and soft-tissue tumor pathologist (JVMGB) according to the 2013 World Health Organization classification. For 10 dedifferentiated chondrosarcomas from the validation cohort, IDH1 and IDH2 mutation analysis was performed in diagnostic setting. All the specimens were anonymized and handled according to the ethical guidelines described in the Code for Proper Secondary Use of Human Tissue in the Netherlands of the Dutch Federation of Medical Scientific Societies.

\section{Immunohistochemistry and Scoring}

Four- $\mu$ m sections were deparaffinized with xylene and rehydrated in graded concentrations of ethanol. Endogenous peroxidase was blocked in $0.3 \% \mathrm{H}_{2} \mathrm{O}_{2}$ solution and microwave antigen retrieval was 
performed in Tris-EDTA pH 9.0 or Citrate $\mathrm{pH} 6.0$ as described in Table 1. For PD-L1 staining, the clone E1L3N (Cell Signaling, Danvers, MA, USA) was used. According to standard laboratory methods, ${ }^{24}$ primary antibodies were diluted in PBS/bovine serum albumin $1 \%$ and incubated overnight at $4{ }^{\circ} \mathrm{C}$, then detected using poly-HRP (ImmunoLogic) and visualized with a $\mathrm{DAB}+$ substrate chromogen system (Dako). Finally, slides were counterstained with hematoxylin, dehydrated and mounted using CV Mount (Leica Microsystems). Tonsils were used as positive controls and primary antibodies were omitted for the negative controls.

Tissue microarrays and whole sections were evaluated and scored independently by two observers (MK and JVMGB or AHGC), blinded to clinicopathological data. For dedifferentiated chondrosarcoma, the well-differentiated and the dedifferentiated components were scored separately. In case of discrepancies, the slide was reviewed to reach a consensus. Extent of PD-L1 staining (1: $<1 \%, 2: 1-49 \%$ and $3: \geq 50 \%)$ was assessed and PD-L1 positivity was defined as $\geq 1 \%$ of tumor cells or immune cells showing a membranous PD-L1 staining of any intensity, according to published data. ${ }^{19}$ The number of CD3-positive cells was quantified manually on a Zeiss microscope in five representative $\times 40$ high-power fields (HPF). Adapted from the landmark paper on prognostic significance of tumor-infiltrating lymphocytes, the degree of $\mathrm{T}$ cells was graded as low if $\leq 5 \mathrm{~T}$ cells/HPF or high if $>5$ $\mathrm{T}$ cells/HPF. ${ }^{25}$ Because of heterogeneity, both intratumoral and peritumoral area of the tumor were scored separately when possible. In tumors with high T-cell infiltrate, PD-1 expression was assessed on $\mathrm{T}$ cells and was considered positive if a membranous staining could be seen. HLA class I expression (HCA2, HC10 and $\beta 2$-microglobulin staining) was categorized as negative/focal weak (tumor cells negative or focally and weakly positive with positive internal controls), heterogeneous (if negative as well as positive regions could be found on the same slide) or positive (moderate or strong staining in the whole tumor). Macrophage infiltrate was scored semiquantitatively on whole section as low or high using the cutoff of 20\% CD14-positive cells. Specimens for which loss of tissue occurred during the staining procedure were not included in the analysis.

\section{Immunofluorescent Staining and Scoring}

Tumor-infiltrating lymphocytes were characterized with triple immunofluorescent staining of CD3-CD8FoxP3 and tumor-associated macrophages with double immunofluorescence of CD14-CD163, both in nine patients (six primary tumors and three not matched metastasis). After antigen retrieval using Tris-EDTA buffer pH 9.0, the combination of primary antibodies were incubated overnight at $4{ }^{\circ} \mathrm{C}$ (see Table 1). The next day, isotype-specific secondary antibodies labeled with Alexa fluorochromes were

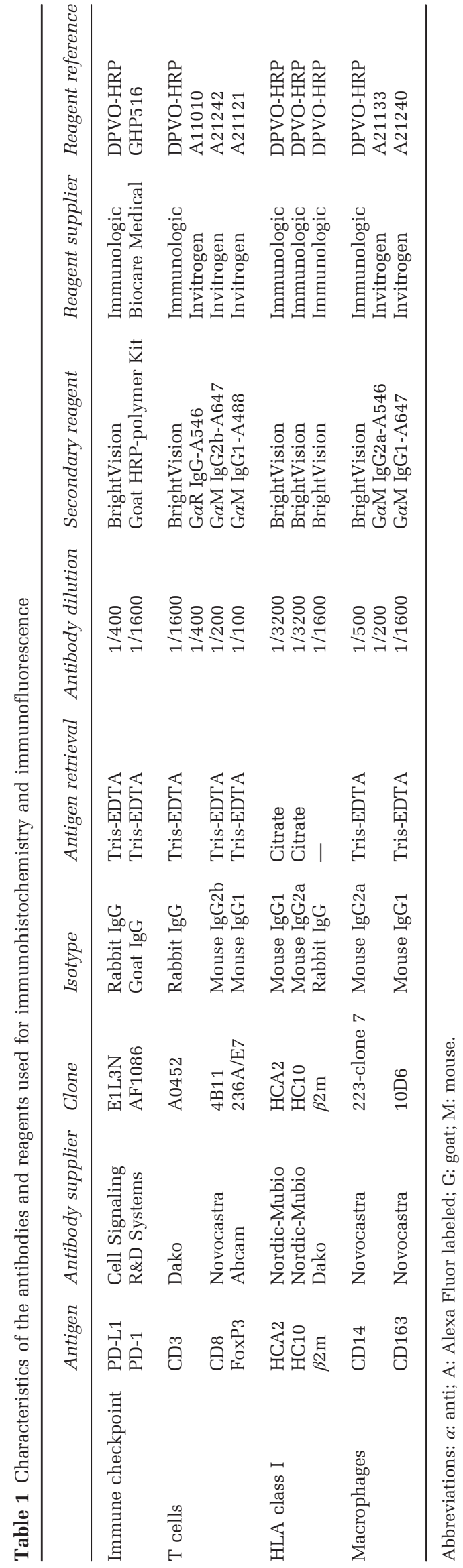


added during $1 \mathrm{~h}$ at room temperature. Then the slides were mounted using Vectashield mounting medium containing DAPI (Vector Laboratories). Tonsils were used as positive controls and primary antibodies were omitted for the negative controls.

Five randomly selected representative images were acquired at $\times 250$ magnification using a confocal scanning microscope (LMS700, Zeiss) in a multitrack setting with a $\times 25 / 0.80$ Plan-Neofluoar objective. The numbers of $\mathrm{T}$ helper cells $\mathrm{CD} 3^{+} \mathrm{CD}^{-}$ (red), $\mathrm{T}$ cytotoxic cells $\mathrm{CD}^{+} \mathrm{CD}^{+}$(purple) and $\mathrm{T}$ regulatory cells $\mathrm{FoxP}^{+}$(green nuclear staining) were counted using the cell counter plugin of the program Image version 1.48 and represented as an average of these five images. Polarization of the CD14-positive tumor-associated macrophages was evaluated qualitatively by expression of the CD163 marker, which represents tumor-promoting type 2 macrophages (M2). We considered that CD14 ${ }^{+}$ CD163 ${ }^{+}$double-positive cells indicated M2 macrophages while $\mathrm{CD} 14^{+} \mathrm{CD} 163^{-}$cells represented the classically activated M1 macrophages. ${ }^{26}$

\section{Statistical Analysis}

Statistical analyses were performed using the GraphPad Prism software (version 6, Jolla, CA, USA). Correlation of PD-L1 expression with the immune infiltrate and the HLA class I phenotype was analyzed by Spearman's rank correlation coefficient. A log-rank test was used for assessing PD-L1 in relation to overall patient survival. $P$-value $<0.05$ was considered to be statistically significant.

\section{Results}

\section{PD-L1 Expression is Found Exclusively in} Dedifferentiated Chondrosarcoma

In the conventional and rare chondrosarcoma tissue microarrays, PD-L1 expression was specifically detected in $41 \%$ of dedifferentiated chondrosarcomas (9 of the 22), whereas its expression was absent in the remaining subtypes (Table 2). Interestingly, only the dedifferentiated component exhibited PD-L1 positivity as shown in Figures 1a and b.

This finding was confirmed on whole tumor sections in an independent cohort of patients with dedifferentiated chondrosarcoma. PD-L1 expression was positive in 11 of the 21 patients (52\%) for which the dedifferentiated component could be evaluated. For one patient, material from both the primary tumor and a synchronous metastasis was available. PD-L1 expression was negative in the primary dedifferentiated tumor but positive in the subcutaneous metastasis. In 10 patients, PD-L1 positivity was observed mainly on the tumor cell membrane and on a few macrophages. Specifically, the extent of PD-L1 expression on tumor cells ranged from 5 to $100 \%$ ( $5 \%$ for two tumors, $30-40 \%$ for three tumors
Table 2 PD-L1 staining in cartilage tumors

\begin{tabular}{|c|c|c|}
\hline Histology & $\mathrm{n}$ & PDL1+ \\
\hline Conventional TMA & 157 & \\
\hline Osteochondroma & 11 & $0 / 8(0 \%)$ \\
\hline \multicolumn{3}{|c|}{ Peripheral chondrosarcoma } \\
\hline Grade I & 31 & $0 / 25(0 \%)$ \\
\hline Grade II & 11 & $0 / 10(0 \%)$ \\
\hline Grade III & 3 & $0 / 3(0 \%)$ \\
\hline Enchondroma & 9 & $0 / 8(0 \%)$ \\
\hline \multicolumn{3}{|c|}{ Central chondrosarcoma } \\
\hline Grade I & 42 & $0 / 40(0 \%)$ \\
\hline Grade II & 36 & $0 / 28(0 \%)$ \\
\hline Grade III & 14 & $0 / 13(0 \%)$ \\
\hline Rare subtypes TMA & 66 & \\
\hline Clear cell & 20 & $0 / 20(0 \%)$ \\
\hline Mesenchymal & 21 & $0 / 19(0 \%)$ \\
\hline Dedifferentiated & 25 & \\
\hline WD component & 17 & $0 / 17(0 \%)$ \\
\hline DD component & 23 & $9 / 22(41 \%)$ \\
\hline Validation cohort & 22 & \\
\hline Dedifferentiated & 22 & \\
\hline WD component & 15 & $0 / 15(0 \%)$ \\
\hline DD component & 22 & $11 / 21(52 \%)$ \\
\hline
\end{tabular}

and $\geq 50 \%$ in the five remaining tumors $(50,60,70$, 70 and $95 \%)$ ). Heterogeneous staining was observed in most of PD-L1-positive tumors except the three tumors with higher PD-L1 expression $(\geq 70 \%)$. For tumors with lower PD-L1 expression (5\%), PD-L1 staining was detected mainly at the periphery, at the interface with normal cartilage, the well-differentiated component or stroma. In one patient, PD-L1 expression was clearly restricted to tumor-associated macrophages (Supplementary Figure S1). To exclude false negative results owing to decalcification during standard tissue preparation, the effect of this procedure on PD-L1 immunostaining was assessed in tonsil tissue. No difference was observed after EDTA or formic acid treatment (Supplementary Figure S2).

We noticed a median overall survival of 10 months for patients with PD-L1-positive dedifferentiated chondrosarcoma and 19 months for patients with PD-L1-negative tumor, although PD-L1 expression did not significantly relate to overall patient survival (log rank; $P=0.22$ ) (Supplementary Figure S3).

\section{PD-L1 Expression in Dedifferentiated Chondrosarcoma Associates with T-Cell Infiltration}

To explore a potential association between PD-L1 expression by tumor cells and the presence of infiltrating immune cells, we evaluated the amount of $\mathrm{T}$ cells based on CD3 immunohistochemistry. High tumor infiltration by $\mathrm{T}$ cells was significantly correlated with PD-L1 expression on tumor cells (Spearman's rho $=0.53, \quad P=0.014$ ). Furthermore, $\mathrm{T}$ cells commonly co-localized with PD-L1-positive tumor cells (Figures 1c and d). Overall, 10 of the 22 

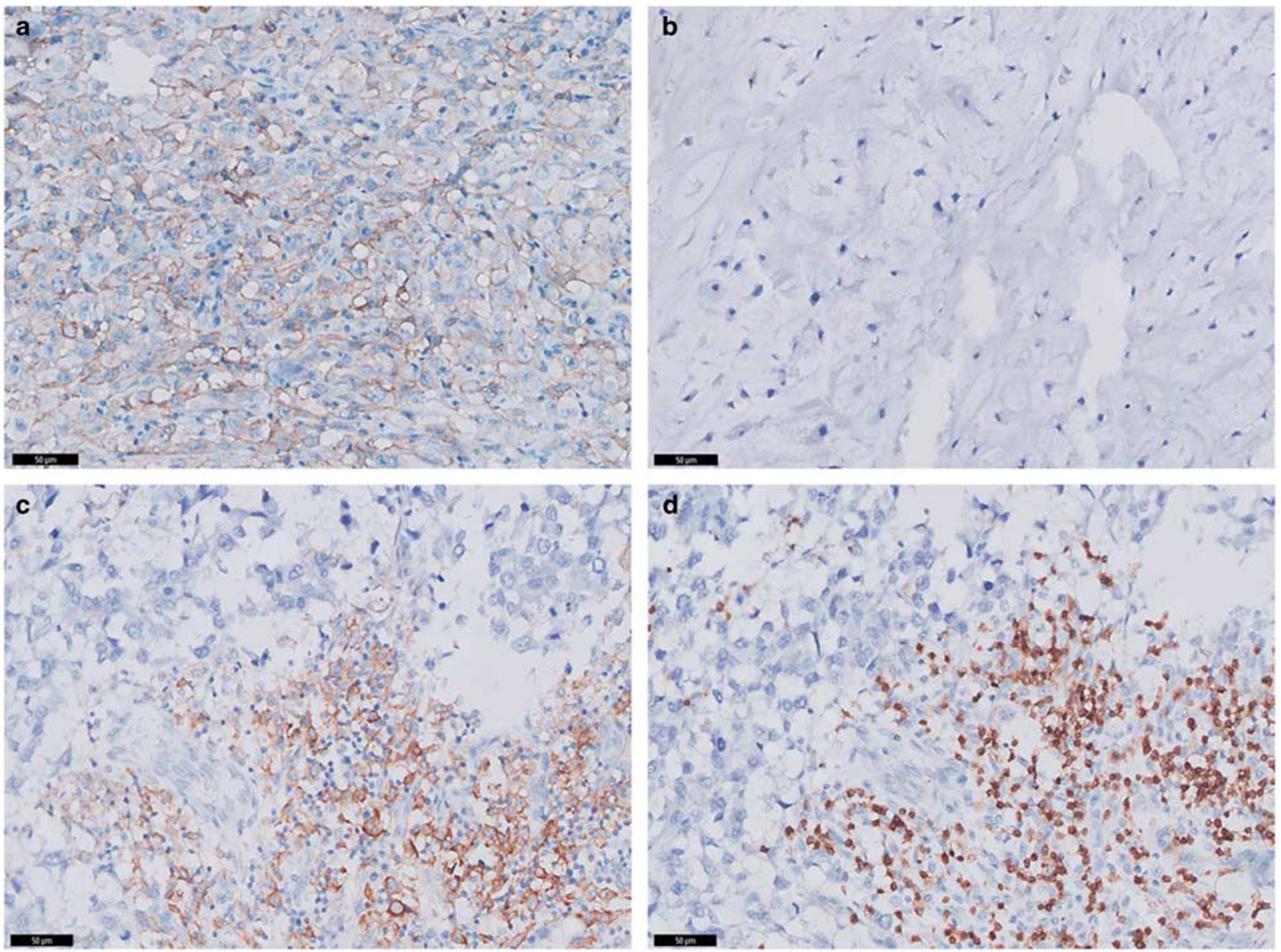

Figure 1 PD-L1 expression is limited to the dedifferentiated component and colocalizes with tumor-infiltrating lymphocytes. Representative images for PD-L1 immunostaining in a dedifferentiated chondrosarcoma with both dedifferentiated (a) and welldifferentiated (b) component. Commonly, a regional colocalization was observed of tumor cells expressing PD-L1 (c) and tumor-infiltrating lymphocytes, characterized by CD3 immunohistochemistry (d) in the dedifferentiated component. Scale bars, $50 \mu \mathrm{m}$.

$(45 \%)$ tumors were highly infiltrated with $\mathrm{T}$ cells. For three patients, we observed a low density of intratumoral T-cell infiltration, whereas higher in the peritumoral area, highlighting the importance of evaluating and scoring a whole tumor section. Nevertheless, these tumors were considered as having low T-cell infiltrate as the absence of intratumoral $\mathrm{T}$ cells would possibly result in lower efficacy of checkpoint blockade therapy. Similar to the distribution of PD-L1 expression, tumorinfiltrating lymphocytes were only present in the dedifferentiated component and absent in the welldifferentiated parts. As PD-L1 is a ligand for the PD-1 receptor expressed in $\mathrm{T}$ cells, we assessed $\mathrm{PD}-1$ expression in tumors with high T-cell infiltration $(n=8)$. In all tumors except one, a positive membranous PD-1 expression was found on $\mathrm{T}$ cells (Supplementary Figure S4).

To further characterize the T-cell infiltrate in these dedifferentiated chondrosarcomas, a triple immunofluorescent staining of CD3-CD8-FoxP3 was performed on six primary tumors and three metastases (Figures 2a and b). $\mathrm{CD}^{+} \mathrm{CD}^{-}$cells were identified as T helper cells (presumably composed of $\mathrm{CD}^{+}$and $\gamma \delta$ T cells), $\mathrm{CD}^{+}{ }^{+} \mathrm{CD} 8^{+}$cells characterized $\mathrm{T}$ cytotoxic cells and the specific transcription factor FoxP3 was used to detect T regulatory cells. Overall, $\mathrm{CD}^{+}{ }^{+} \mathrm{CD}^{+}{ }^{+}$cytotoxic $\mathrm{T}$ cells represented $58 \%$ of the tumor-infiltrating lymphocytes in both primary tumors and metastases. T regulatory cells were more frequent in metastases than in primary tumors (18 versus $4 \%$ of total tumor-infiltrating lymphocytes) and were all $\mathrm{CD}^{+} \mathrm{FoxP}^{+}$double-positive cells. No $\mathrm{CD}^{+}{ }^{+} \mathrm{FoxP}^{+}$cells were found. Finally, the proportion of $\mathrm{T}$ helper cells $\mathrm{CD}^{+} \mathrm{CD}^{-}$was $38 \%$ in primary tumors versus $24 \%$ in metastases (Figure 2c).

IDH mutation status was available for 10 dedifferentiated chondrosarcomas. Five tumors harbored an IDH1 mutation, one had an IDH2 mutation and four tumors were of wild type. No differences regarding T-cell infiltrate or PD-L1 expression were observed between $I D H$ mutant and $I D H$ wild type. 


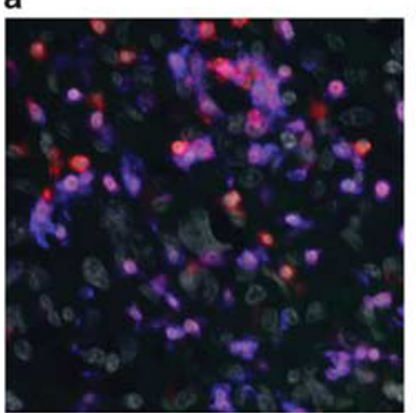

d

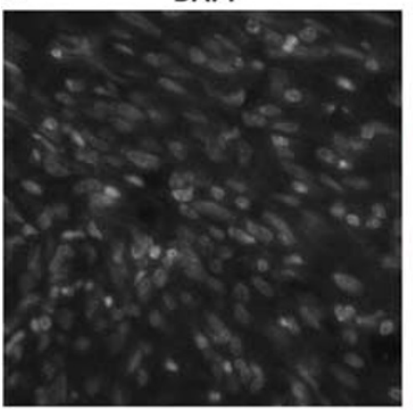

b

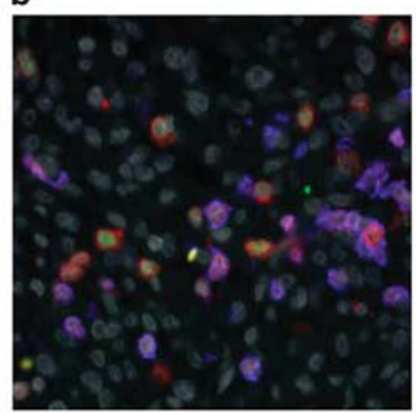

CD14

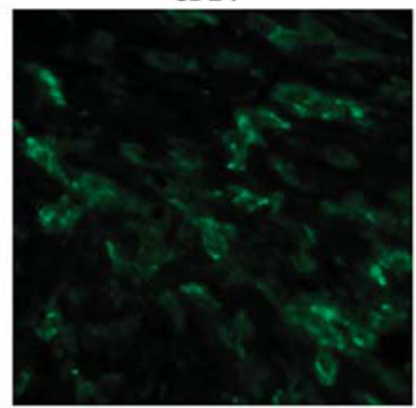

C

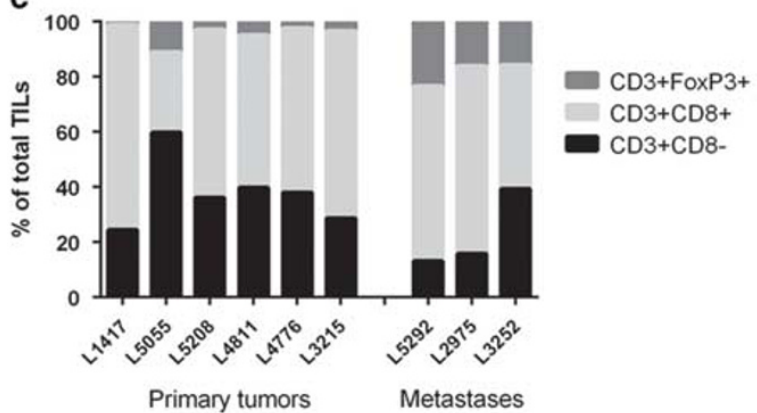

CD163

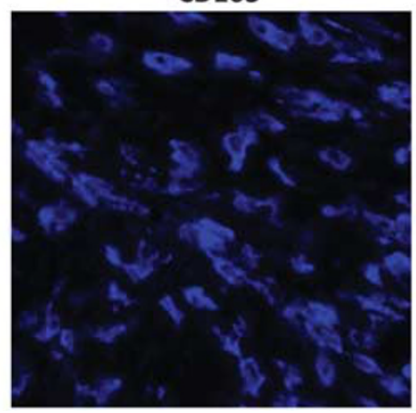

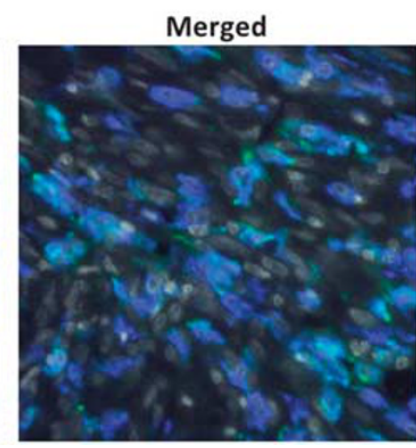

Figure 2 Characterization of tumor-infiltrating lymphocytes and tumor-associated macrophages in dedifferentiated chondrosarcoma. Representative images for tumor-infiltrating lymphocytes in primary tumors (a) and in metastases (b). Antibodies against CD3 (red), CD8 (blue) and FoxP3 (green) were used to identify T helper cells $\left(\mathrm{CD} 3^{+} \mathrm{CD} 8^{-}\right)$, cytotoxic $\mathrm{T}$ cells $\left(\mathrm{CD} 3^{+} \mathrm{CD} 8^{+}\right)$and $\mathrm{T}$ regulatory cells $\left(\mathrm{FoxP} 3^{+}\right)$. In both primary tumors and metastases, around $60 \%$ of tumor-infiltrating lymphocytes were cytotoxic CD3 ${ }^{+} \mathrm{CD} 8^{+} \mathrm{T}$ cells $(\mathbf{c})$. Furthermore, a higher percentage of $\mathrm{FoxP}^{+} \mathrm{T}$ regulatory cells was found in metastases (mean 18\%) compared with primary tumors (mean $5 \%$ ). Representative images for tumor-associated macrophages (d). Antibodies against CD14 (green) and CD163 (blue) were used to detect macrophages and characterize their polarization as CD163 represents tumor-promoting type 2 macrophages (M2).

\section{Dedifferentiated Chondrosarcomas Display Different Phenotypes for HLA Class I Expression}

As HLA class I antigen expression is an important determinant for T-cell based immunotherapy, we assessed its expression in dedifferentiated chondrosarcomas by immunohistochemistry. Antibodies against $\beta 2$-microglobulin and classical HLA class I molecules were used (HCA2 and HC10, which recognize heavy chains of HLA-A and HLA-B/C, respectively). In the dedifferentiated component, positive membranous and cytoplasmic expression of $\beta 2$-microglobulin was found in all tumors. Expression of HCA2 and HC10 were mostly detected at the membrane of tumor cells. Positive and diffuse expression $(n=9)$, heterogeneous expression $(n=5)$ or weak/negative expression $(n=2)$ was present for both markers. In four patients, we observed a weak or negative HCA2 expression associated with positive HC10 expression, reflecting a selective loss of HLA-A expression. These different patterns for HLA class I expression are illustrated in Figure 3. Altogether, defects in HLA class I expression were present in 11 of the 20 evaluated tumors (55\%). Expression of $\beta 2$-microglobulin, HCA2 and HC10 in the evaluable well-differentiated cartilaginous areas of the tumors was mainly heterogeneous with coexistence of both positive and negative cells.
Positive HLA class I expression was significantly associated with high T-cell infiltrate and PD-L1 expression on tumor cells (Spearman's rho $=0.503$, $P=0.024)$.

\section{The Dedifferentiated Component is Highly Infiltrated by Immunosuppressive Macrophages}

Based on the presence of PD-L1-positive macrophages in some tumors, we evaluated the macrophage infiltrate in dedifferentiated chondrosarcomas using CD14 immunohistochemistry. On 20 evaluable tumors, 17 were highly infiltrated by tumor-associated macrophages (85\%). Consistent with PD-L1 expression and the presence of tumor-infiltrating lymphocytes, macrophages were found only in the dedifferentiated areas and not in the well-differentiated component. Immunofluorescent staining with CD14 and CD163 performed on nine tumors (six primary tumors and three metastases) demonstrated that all the CD14-positive cells were also CD163 positive, a marker of alternatively activated and immunosuppressive macrophages (Figure 2d). We also observed some CD14-CD163 ${ }^{+}$cells.

Statistical analysis showed no significant correlation between macrophage and T-cell infiltration (Spearman's rho $=0.38, P=0.098$ ). However, the three tumors with a low number of tumor-associated 

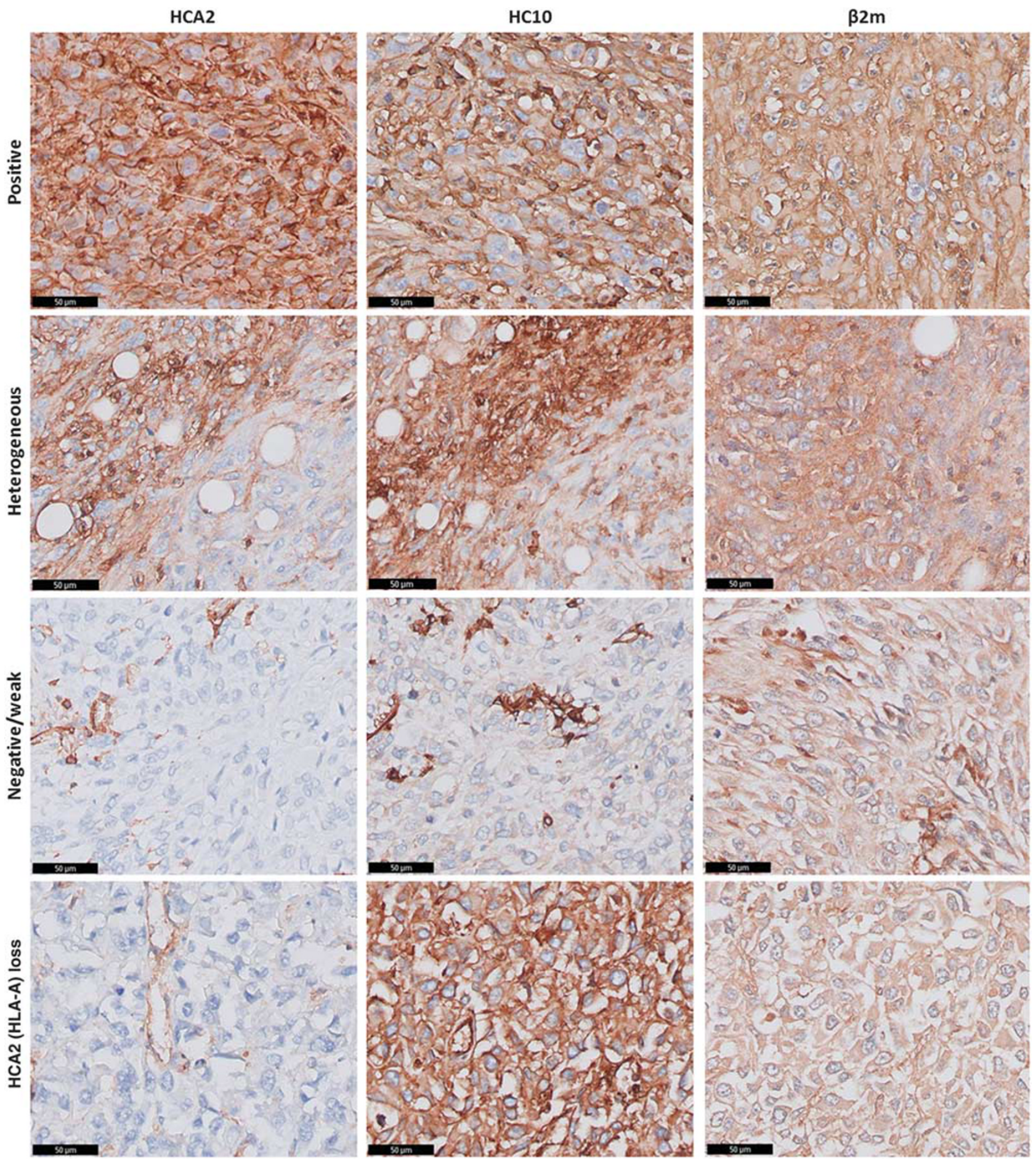

Figure 3 Different HLA class I phenotypes in dedifferentiated chondrosarcomas. Representative images for the HLA class I evaluated by immunohistochemistry with HCA2 (for HLA-A), HC10 (for HLA-B/C) and $\beta 2$-microglobulin antibodies. Similar expression of HLA-A and $\mathrm{B} / \mathrm{C}$ on tumor cells was often observed and categorized as positive $(n=9)$, heterogeneous $(n=5)$ or negative/weak $(n=2)$ with appropriate positive internal controls (endothelial cells or T cells). Four tumors showed selective loss of HLA-A with positive expression of HLA-B/C. B2-microglobulin was positive in all tumors. Scale bars, $50 \mu \mathrm{m}$.

macrophages were also poorly infiltrated by $\mathrm{T}$ cells. The macrophage infiltrate was quite homogeneous within the dedifferentiated component and colocalization with PD-L1-positive tumor cells was not evident.

\section{Discussion}

The growing interest in cancer immunotherapy is reaching the sarcoma field, and we are currently at the point of identifying which sarcomas are 
amenable to immune checkpoint blockers. Those with high immune infiltrate and high mutational load or complex karyotype appear to be the best candidates. ${ }^{10}$ To date, no studies have evaluated the PD-1/PD-L1 axis in chondrosarcoma. The first clinical data on the immune checkpoint blockade strategy in these patients will be shortly provided with the ongoing phase II trial evaluating the anti PD-1 antibody pembrolizumab in advanced bone and soft-tissue sarcomas. ${ }^{27}$ Our screening of PD-L1 expression by immunohistochemistry in a large cohort of chondrosarcoma patients revealed positive staining exclusively in the dedifferentiated subtype. Overall, PD-L1 expression was detected in $41 \%$ of the dedifferentiated chondrosarcomas. To validate this finding, whole tumor sections of an independent cohort of 22 patients with dedifferentiated chondrosarcoma were assessed for PD-L1 expression, which showed positive staining in $52 \%$ of the tumors. Overall, PD-L1 expression on tumor cells ranged from $<1 \%$ (considered negative) to almost $100 \%$. This PD-L1 positivity was associated with high T-cell infiltrate, reflecting an immune-active microenvironment.

Dedifferentiated chondrosarcoma is a highly malignant sarcoma and chemotherapy or radiation therapy do not improve prognosis for inoperable metastatic patients owing to resistance. So far, no potential targets for therapy could be identified. Interestingly, we found that PD-L1 positivity was limited to the dedifferentiated area of the tumors, which was also enriched for immune-infiltrating lymphocytes and immunosuppressive macrophages. The well-differentiated components evaluated in our cohort were all poorly infiltrated with immune cells, which may be explained by its abundant cartilaginous matrix and poor vascularization, thereby possibly limiting accessibility to tumor cells. Hence, our data suggest that immune checkpoint inhibitors may be effective to treat the dedifferentiated component of dedifferentiated chondrosarcoma, while the effect on the less aggressive cartilaginous welldifferentiated component is expected to be minimal or even absent. Our results are in line with the fact that additional genetic changes occur in the anaplastic component of dedifferentiated chondrosarcoma, which may lead to higher numbers of mutations in this part of the tumor and thus occurrence of neoantigens. ${ }^{16,28}$ We have learned from epithelial malignancies that tumors with high mutational load show strong T-cell responses and appear to be particularly sensitive to immunotherapy, such as immune checkpoint blockade. ${ }^{29}$ This was also shown in animal models of osteosarcoma, a sarcoma known for its high genomic instability. ${ }^{30}$ In dedifferentiated chondrosarcoma, mutations in IDH1 or IDH2 are present in around half of the tumors, in both components. ${ }^{5}$ In our series, information regarding $I D H$ mutation status was available for 10 tumors. The presence of an $I D H$ mutation was not associated with the expression of PD-L1 or a specific immune profile
(HLA class I expression, amount of T-cell and macrophage infiltrate). A previous study reported a spontaneous peripheral T-cell response against IDH1-mutated gliomas but did not analyze the immune microenvironment of these tumors. ${ }^{31}$ Larger series are needed before any conclusions on the role of $I D H$ in antitumor immunity can be drawn in chondrosarcoma.

Characterization of the sarcoma microenvironment is an essential prerequisite to understand the role of the immune system in these mesenchymal tumors and to assess the feasibility of immunotherapy. As patients with chondrosarcoma usually do not receive neoadjuvant treatment, the primary tumors reflect the natural host immune response and the natural tumor microenvironment. PD-L1-positive dedifferentiated chondrosarcoma exhibited a significantly increased number of $\mathrm{CD}^{+}$and $\mathrm{CD}^{+} \mathrm{T}$ cells that colocalized with PD-L1-positive tumor cells, supporting an adaptive mechanism of immune escape, as described first in melanoma. ${ }^{32,33}$ In addition, HLA class I loss of expression is another possible immune escape mechanism employed by tumor cells to avoid T-cell recognition. ${ }^{34}$ Overall, we observed defects in HLA class I expression in 55\% of the dedifferentiated chondrosarcomas, which is in accordance with the recently reported data. ${ }^{35}$ Mutations in $\beta 2$-microglobulin is one of the mechanisms related to HLA class I defects but do not seem to be involved in our series as the expression of $\beta 2$-microglobulin was found in all tumors. However, we did not observe a complete loss of HLA class I but rather weak or heterogeneous expression. Little is known about HLA class I expression in normal cartilage and cartilaginous tumors, and future functional studies should determine mechanisms and potential reversibility of low HLA class I expression. HLA class I expression on tumor cells correlated with high T-cell infiltrate and the presence of PD-L1 expression, which emphasizes the close interrelationship among these immune parameters. Thus, detection of HLA class I expression might be due to the presence of an inflammatory environment while in the negative/focal weak cases we cannot determine whether HLA class I is lost or whether its expression is not stimulated by the immune system. These results also demonstrate that some dedifferentiated chondrosarcomas are clearly more immunogenic than others. Previous preclinical studies identified some immunogenic targets in chondrosarcoma, such as the cancer-testis antigens MAGE-A3, NY-ESO-1/ LAGE-1 and PRAME, which were able to induce a specific cytotoxic T-cell response in the context of HLA class I expression. ${ }^{36,37}$ NY-ESO-1 expression was observed not only on dedifferentiated but also on conventional chondrosarcoma. ${ }^{38}$

Importantly, the matched primary tumor and subcutaneous metastasis evaluated in one patient displayed discordant PD-L1 expression. Also, the higher proportion of $\mathrm{FoxP}^{+} \mathrm{T}$ regulatory cells in metastases compared with primary tumors may 
suggest a specific role of these immunosuppressive cells in later stages of the disease. These observations emphasize the dynamics of the immune response and the tumor heterogeneity. Whole section evaluation seems more appropriate to assess these immune markers, as illustrated by the heterogeneous pattern of T-cell infiltration and HLA class I expression. Only the macrophage infiltrate appeared homogeneous within the dedifferentiated component. Significance and functions of these immunosuppressive CD163-positive macrophages in the dedifferentiated area need to be further elucidated.

Development of appropriate biomarkers to select patients who are likely to benefit from anti-PD-1 or anti-PD-L1 therapies remains a challenge as an important proportion of patients fail to respond to these treatments. Depending on the tumor type, some data suggest that PD-L1 expression on pretreatment specimens is associated with higher response rates. ${ }^{9,16}$ However, the use of various PD-L1 clones with different detection methods and interpretation makes literature confusing and challenging to draw conclusions. In sarcoma patients, for instance, different expression patterns of PD-L1 are observed in soft-tissue sarcomas when comparing two studies. ${ }^{39,40} \mathrm{Kim}$ et $\mathrm{al}^{39}$ reported $58 \%$ of PD-L1positive tumors (clone $\mathrm{H}-130$, Santa Cruz), which correlated with overall survival, while D'Angelo et $a l^{40}$ found uncommon PD-L1 expression on tumor cells not related with patient survival (clone $5 \mathrm{H}-1$, DAKO assay). In the future, efforts should be made to standardize PD-L1 evaluation. In osteosarcoma, PD-L1 staining was evaluated by immunofluorescence (monoclonal antibody from Abcam), indicating PD-L1 expression in $75 \%$ of metastatic lesions but not on primary tumors. ${ }^{41}$ Heterogeneity not only between sarcoma subtypes but also within tumors is another issue to take into consideration. Thus evaluation of PD-L1 expression within each histological subtype seems more relevant to estimate the prevalence and predictive value of this immune marker.

In our study, the presence of immune-infiltrating cells, notably PD-1-positive $\mathrm{T}$ cells, together with HLA class I expression in the PD-L1-positive dedifferentiated chondrosarcomas suggests that these patients may be excellent candidates for PD-1/PD-L1-targeted therapies. Their inclusion in clinical trials would help (1) to assess a potential benefit for patients with dedifferentiated chondrosarcoma and (2) to elucidate whether the PD-L1 expression observed in half of the tumors is predictive for objective response to agents targeting the PD-1/PD-L1 pathway.

\section{Acknowledgments}

We thank Dorien van der Geest and Jolieke van Oosterwijk for constructing the conventional chondrosarcoma tissue microarray, Danielle Meijer for constructing the rare chondrosarcoma subtypes tissue microarray and all the medical centers that provided tissue for these two tissue microarrays: Nuffield Department of Orthopedic Surgery, University of Oxford, Oxford, UK; Laboratory of Oncologic Research, Rizzoli Orthopedic Institute, Bologna, Italy; Department of Pathology, Copenhagen University Hospital, Copenhagen, Denmark; and Department of Pathology, Medical University of Graz, Graz, Austria. We thank Inge $\mathrm{H}$ Briaire-de Bruijn for expert technical assistance with the tissue microarrays and Ekaterina S Jordanova and Brendy E van den Akker for help with immunofluorescent procedures. We also thank Yvonne de Yong and Arnoud Boot for discussions. Marie Kostine was supported in part by a grant from the Université de Bordeaux/Centre Hospitalier Universitaire de Bordeaux, France.

\section{Disclosure/conflict of interest}

The authors declare no conflict of interest.

\section{References}

1 Hogendoorn PC, Bovée JV, Nielsen GP, et al. Chondrosarcoma (grade I-III), including primary and secondary variants and periosteal chondrosarcoma, dedifferentiated chondrosarcoma, mesenchymal chondrosarcoma and clear cell chondrosarcoma. In: Fletcher CDM, Bridge JA, Hogendoorn PC, et al (eds). WHO Classification of Tumours of Soft Tissue and Bone. 4th edn. IARC Press: Lyon, France, 2013, pp 264-274.

2 Amary MF, Bacsi K, Maggiani F, et al. IDH1 and IDH2 mutations are frequent events in central chondrosarcoma and central and periosteal chondromas but not in other mesenchymal tumours. J Pathol 2011;224: 334-343.

3 Bovée JV, Hogendoorn PC, Wunder JS, et al. Cartilage tumours and bone development: molecular pathology and possible therapeutic targets. Nat Rev Cancer 2010;10:481-488.

4 Gelderblom H, Hogendoorn PC, Dijkstra SD, et al. The clinical approach towards chondrosarcoma. Oncologist 2008;13:320-329.

5 Meijer D, de Jong D, Pansuriya TC, et al. Genetic characterization of mesenchymal, clear cell, and dedifferentiated chondrosarcoma. Genes Chromosomes Cancer 2012;51:899-909.

6 Hodi FS, O'Day SJ, McDermott DF, et al. Improved survival with ipilimumab in patients with metastatic melanoma. N Engl J Med 2010;363:711-723.

7 Weber JS, D'Angelo SP, Minor D, et al. Nivolumab versus chemotherapy in patients with advanced melanoma who progressed after anti-CTLA-4 treatment (CheckMate 037): a randomised, controlled, open-label, phase 3 trial. Lancet Oncol 2015;16:375-384.

8 Robert C, Schachter J, Long GV, et al. Pembrolizumab versus ipilimumab in advanced melanoma. N Engl J Med 2015;372:2521-2532.

9 Borghaei H, Paz-Ares L, Horn L, et al. Nivolumab versus docetaxel in advanced nonsquamous non-smallcell lung cancer. N Engl J Med 2015;373:1627-1639. 
10 Lim J, Poulin NM, Nielsen TO. New strategies in sarcoma: linking genomic and immunotherapy approaches to molecular subtype. Clin Cancer Res 2015;21:4753-4759.

11 Kim C, Kim EK, Han JW, et al. Clinical pattern and implication of PD-L1 expression in soft-tissue sarcoma (ASCO Meeting 2015). J Clin Oncol 2015;33 (Suppl; abstr 10565).

12 Topalian SL, Drake CG, Pardoll DM. Immune checkpoint blockade: a common denominator approach to cancer therapy. Cancer Cell 2015;27:450-461.

13 Snyder A, Makarov V, Merghoub T, et al. Genetic basis for clinical response to CTLA-4 blockade in melanoma. N Engl J Med 2014;371:2189-2199.

14 Rizvi NA, Hellmann MD, Snyder A, et al. Cancer immunology. Mutational landscape determines sensitivity to PD-1 blockade in non-small cell lung cancer. Science 2015;348:124-128.

15 Tumeh PC, Harview CL, Yearley JH, et al. PD-1 blockade induces responses by inhibiting adaptive immune resistance. Nature 2014;515:568-571.

16 Tarpey PS, Behjati S, Cooke SL, et al. Frequent mutation of the major cartilage collagen gene COL2A1 in chondrosarcoma. Nat Genet 2013;45:923-926.

17 Meng X, Huang Z, Teng F, et al. Predictive biomarkers in PD-1/PD-L1 checkpoint blockade immunotherapy. Cancer Treat Rev 2015;41:868-876.

18 Robert C, Long GV, Brady B, et al. Nivolumab in previously untreated melanoma without BRAF mutation. N Engl J Med 2015;372:320-330.

19 Garon EB, Rizvi NA, Hui R, et al. Pembrolizumab for the treatment of non-small-cell lung cancer. N Engl J Med 2015;372:2018-2028.

20 Herbst RS, Baas P, Kim DW, et al. Pembrolizumab versus docetaxel for previously treated, PD-L1-positive, advanced non-small-cell lung cancer (KEYNOTE-010): a randomised controlled trial. Lancet 2015;387:1540-1550.

21 Garrido F, Aptsiauri N, Doorduijn EM, et al. The urgent need to recover MHC class I in cancers for effective immunotherapy. Curr Opin Immunol 2016;39: $44-51$.

22 van Oosterwijk JG, van Ruler MA, Briaire-de Bruijn IH, et al. Src kinases in chondrosarcoma chemoresistance and migration: dasatinib sensitises to doxorubicin in TP53 mutant cells. Br J Cancer 2013;109:1214-1222.

23 van Oosterwijk JG, Meijer D, van Ruler MA, et al. Screening for potential targets for therapy in mesenchymal, clear cell, and dedifferentiated chondrosarcoma reveals Bcl-2 family members and TGF $\beta$ as potential targets. Am J Pathol 2013;182:1347-1356.

24 Baranski Z, Booij TH, Cleton-Jansen AM, et al. Avenmediated checkpoint kinase control regulates proliferation and resistance to chemotherapy in conventional osteosarcoma. J Pathol 2015;236:348-359.

25 Zhang L, Conejo-Garcia JR, Katsaros D, et al. Intratumoral $\mathrm{T}$ cells, recurrence, and survival in epithelial ovarian cancer. N Engl J Med 2003;348:203-213.

26 Heusinkveld M, van der Burg SH. Identification and manipulation of tumor associated macrophages in human cancers. J Transl Med 2011;9:216.

27 Sarcoma Alliance for Research through Collaboration. SARC028: A phase II study of the anti-PD1 antibody
Pembrolizumab (MK-3475) in patients with advanced sarcomas. In: ClinicalTrials.gov [Internet]. Bethesda (MD): National Library of Medicine (US). 2000- [cited 2016 Feb 25]. Available from: http://clinicaltrials.gov/ show/NCT02301039.

28 Bovée JV, Cleton-Jansen AM, Rosenberg C, et al. Molecular genetic characterization of both components of a dedifferentiated chondrosarcoma, with implications for its histogenesis. J Pathol 1999;189:454-462.

29 Schumacher TN, Schreiber RD. Neoantigens in cancer immunotherapy. Science 2015;348:69-74.

30 Lussier DM, Johnson JL, Hingorani P, et al. Combination immunotherapy with $\alpha$-CTLA- 4 and $\alpha$-PD-L1 antibody blockade prevents immune escape and leads to com-

plete control of metastatic osteosarcoma. J Immunother Cancer 2015;3:21.

31 Schumacher T, Bunse L, Pusch S, et al. A vaccine targeting mutant IDH1 induces antitumour immunity. Nature 2014;512:324-327.

32 Taube JM, Anders RA, Young GD, et al. Colocalization of inflammatory response with B7-h1 expression in human melanocytic lesions supports an adaptive resistance mechanism of immune escape. Sci Transl Med 2012;4:127-137.

33 Pardoll DM. The blockade of immune checkpoints in cancer immunotherapy. Nat Rev Cancer 2012;12: 252-264.

34 Campoli M, Ferrone S. HLA antigen and NK cell activating ligand expression in malignant cells: a story of loss or acquisition. Semin Immunopathol 2011;33: 321-334.

35 Nota S, Sabbatino F, Nielsen P, et al. Human Leukocyte Antigen (HLA) Expression and Immunological Events in Chondrosarcoma. In: International Society of Limb Salvage (ISOLS) and Musculoskeletal Tumor Society (MSTS) annual meeting; 2015 Oct 6-10; Orlando. Rosemont (IL): ISOLS-MSTS; 2015. Abstract no. 11468.

36 Bluman EM, Coulie PG, Xiaojuan S, et al. Lysis of human chondrosarcoma cells by cytolytic T lymphocytes recognizing a MAGE-A3 antigen presented by HLA-A1 molecules. J Orthop Res 2007;25:678-684.

37 Pollack SM, Li Y, Blaisdell MJ, et al. NYESO-1/ LAGE-1s and PRAME are targets for antigen specific $\mathrm{T}$ cells in chondrosarcoma following treatment with 5Aza-2-deoxycitabine. PLoS One 2012;7:e32165.

38 Endo M, de Graaff MA, Ingram DR, et al. NY-ESO-1 (CTAG1B) expression in mesenchymal tumors. Mod Pathol 2015;28:587-595.

39 Kim JR, Moon YJ, Kwon KS, et al. Tumor infiltrating PD1-positive lymphocytes and the expression of PD-L1 predict poor prognosis of soft tissue sarcomas. PLoS One 2013;8:e82870.

40 D'Angelo SP, Shoushtari AN, Agaram NP, et al. Prevalence of tumour-infiltrating lymphocytes and PD-L1 expression in the soft tissue sarcoma microenvironment. Hum Pathol 2015;46:357-365.

41 Lussier DM, O’Neill L, Nieves LM, et al. Enhanced T-cell immunity to osteosarcoma through antibody blockade of PD-1/PD-L1 interactions. J Immunother 2015;38:96-106.

Supplementary Information accompanies the paper on Modern Pathology website (http://www.nature.com/ modpathol) 